\title{
Fully 2D quantum-mechanical simulation of nanoscale MOSFETs
}

\author{
A. Pirovano, A. L. Lacaita, and A. S. Spinelli $\ddagger$ \\ DEI, Politecnico di Milano, P.zza L. da Vinci 32, 20133 Milano, Italy \\ ${ }^{\ddagger}$ Dip. di Scienze CCFFMM, Univ. dell'Insubria and INFM, Unità di Como \\ via Valleggio II, 22100 Como, Italy
}

\begin{abstract}
We present results of fully 2D quantum-mechanical (QM) simulations of nanoscale MOSFET's. The validity of semiclassical transport models are first discussed. Then, QM effects on threshold voltage, subthreshold slope and short-channel performances are addressed. We show that QM effects significantly affect device performances in the nanoscale range.
\end{abstract}

\section{Introduction}

Careful evaluation of quantum mechanical (QM) effects has become a compelling issue in the design of aggressively scaled MOSFET's, where the confinement in the direction normal to the $\mathrm{Si} / \mathrm{SiO}_{2}$ interface affects important device parameters such as threshold voltage and gate capacitance. As the channel length is reduced below $50 \mathrm{~nm}$, QM effects in the source-to-drain direction become also relevant, requiring a $2 \mathrm{D}$ treatment of the problem. To this aim, the effects of both the 2D QM charge distribution and QM charge transport should be assessed. However, recent studies suggested that semiclassical models for transport can be effectively used for channel lengths down to $10 \mathrm{~nm}$ or beyond [1,2]. Moreover, several important device parameters such as threshold voltage and subthreshold slope are only marginally affected by the actual transport model used. For these reasons, we have investigated QM effects on the performances of nanoscale MOSFET's relying on a fully 2D self-consistent Schrödinger-Poisson simulator coupled to a semiclassical transport model.

\section{Numerical model}

The inversion layer charge is computed solving the coupled 2D Schrödinger and Poisson equation system, while the transport is self-consistently included according to a drift-diffusion framework. Good convergence properties are obtained using the effective intrinsic density concept [5], where the local $n_{i}$ is modified to account for the QM charge. This approach is also profitably used to resolve the inconsistency between zero QM charge at the boundaries (consequence of the closed-boundary conditions) and non-zero current flow: A classical charge distribution can be effectively recovered in the contact regions by a smoothing procedure on $n_{i}$. This approach results in a highly-effective fully 2D self-consistent QM model, achieving an exponential convergence rate. The accuracy of the solution against grid dimension was verified, as well as the influence of the boundary conditions, by changing the extension of the contact regions. Even if a detailed description of the charge transport was not our goal, a transport model based on the quantum transmitting boundary method [3] was also developed as a benchmark. 


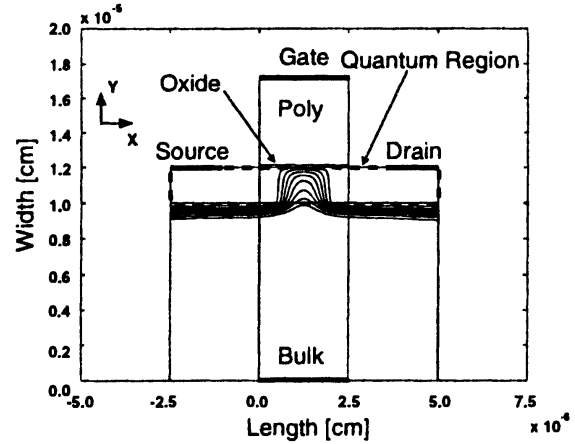

Figure 1: $25 \mathrm{~nm}$ MOSFET structure highlighting contacts, potential contours, and the box region where the $\mathrm{QM}$ charge is calculated.

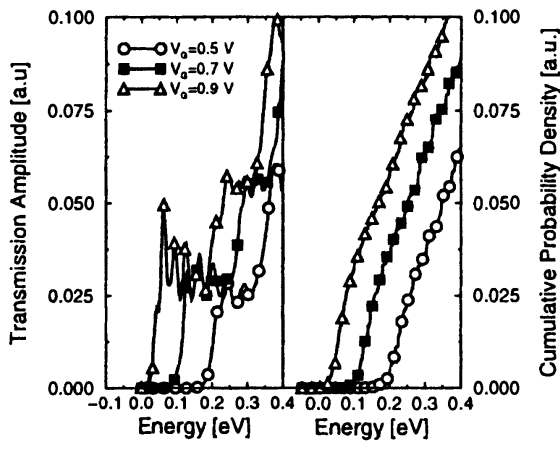

Figure 3: Same as Fig. 2 but in linear scale.

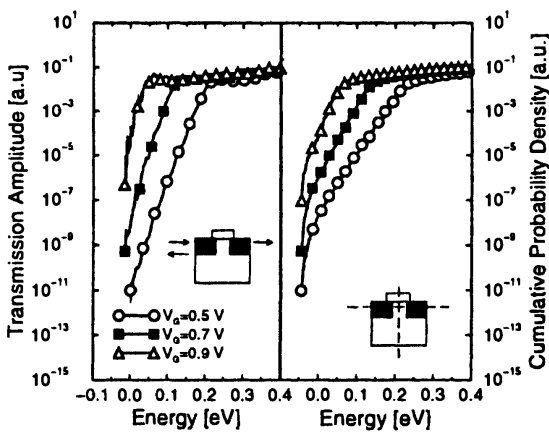

Figure 2: Comparison between TA and CPD at different gate biases for a $25 \mathrm{~nm}$ MOSFET (logarithmic scale).

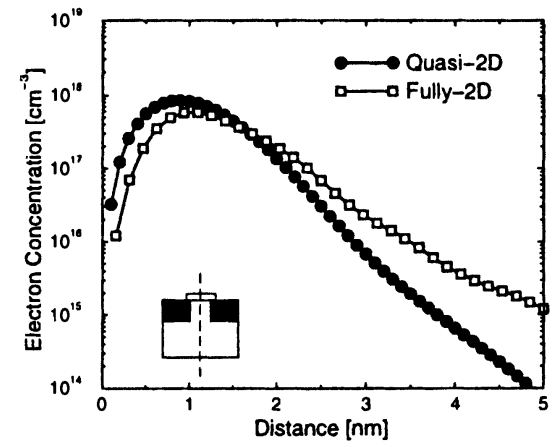

Figure 4: Electron concentration according to a fully-2D and to a quasi-2D QM model.

\section{Impact of quantum transport}

Fig. 1 shows the simulated structure, a $25 \mathrm{~nm} n^{+}$-poly n-MOSFET with an oxide thickness of $1.5 \mathrm{~nm}$ and a uniform channel doping of $2 \times 10^{19} \mathrm{~cm}^{-3}$. The source/drain junction depth is $20 \mathrm{~nm}$, with a lateral abruptness of $1.5 \mathrm{~nm} /$ decade, complying with the requirements for sub-100 nm node technology [4]. Fig. 1 also shows the box region where the QM charge is actually computed, the device contacts, and the potential contours. We have first investigated the impact of the transport model used on the device current: Figs. 2 and 3 show a comparison between the transmission amplitude (TA) for wavefunctions propagating along the channel direction (calculated with the quantum transmitting boundary method) and the cumulative probability density (CPD) in the middle of the channel (computed within the drift-diffusion framework), as a function of the energy and for different gate biases. Note that TA determines the current in a QM transport framework, while CPD is proportional to the current in the semiclassical model. In Fig. 2 TA and CPD are displayed on a logarithmic scale. A good agreement can be noticed, with an exponential growth due to tunneling of the wavefunctions from the contact regions into the channel. When looked on a linear scale (Fig. 3), TA shows a 

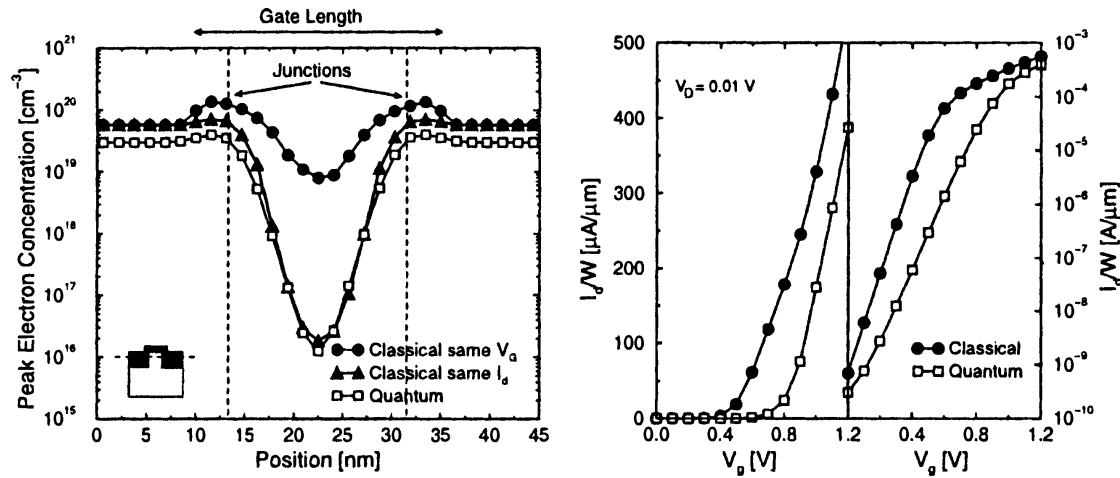

Figure 5: Peak electron concentration along the channel for $\mathrm{QM}$ and classical simulaFigure 6: I-V characteristics at $V_{D}=10 \mathrm{mV}$ for the device in Fig. 1. tions.

step-like dependence in correspondence of the bound levels, while CPD is almost linear. However, these differences do not have any impact on the final result: To obtain the current, both TA and CPD must be weighted by the exponentially decreasing distribution function. Therefore, the main contribution to the current comes from the energy region lying within about $k_{B} T$ from the first quantized level, which corresponds to the onset of TA and CPD. Since in both TA and CPD the onset is the same irrespective of the gate bias, we conclude that these differences will not affect strongly the charge distribution and device current. In the following, we will therefore refer to results obtained with the semiclassical transport model.

\section{Device performances}

Fig. 4 shows a comparison between the QM charge along a section in the middle of the channel computed according to our model and to a quasi-2D QM model, for the same device as in Fig. 1 biased at $V_{G}=0.6 \mathrm{~V}$ and $V_{D}=0.01 \mathrm{~V}$. Non-negligible differences are obtained, demonstrating that a fully-2D treatment of QM effects is essential to reliably assess the charge distribution in nanoscale MOS devices. The peak charge concentration along the channel direction is instead shown in Fig. 5, according to 2D classical and QM calculations. At the same gate bias, QM effects result in less charge in the channel and in the spacer regions, where the confinement is still noticeable. Comparable channel charge is instead obtained at the same output current. However, even in this case, a small difference can be seen in the non-confined source/drain regions. This is due to the vanishing QM charge at the boundary, which determines a "dark space" where the carrier density is reduced compared to the equilibrium value. The classical distribution is in fact exactly recovered at a slightly larger distance from the boundary as well as at the device contacts. The drain current at $V_{D}=10 \mathrm{mV}$ is shown in Fig. 6. A threshold voltage $\left(V_{T}\right)$ shift is apparent, while a comparable transconductance above threshold is obtained. The $V_{T}$ shift $(300 \mathrm{mV})$ is significantly lower than what estimated by quasi-2D QM treatments $(400 \mathrm{mV})$. This discrepancy can be ascribed to the modification in the potential shape induced by the short-channel effects (SCE), and can be captured only with a self-consistent 2D QM model. Moreover, the subthreshold slope 


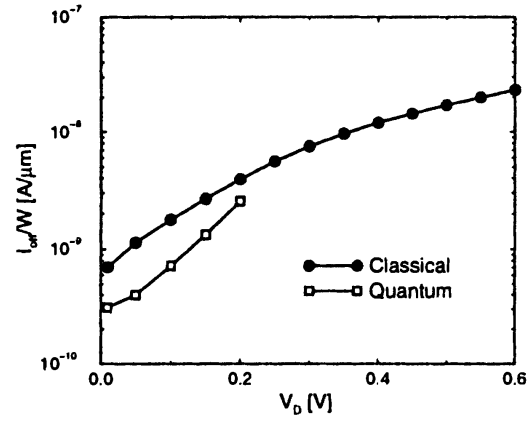

Figure 7: DIBL characteristics (off current) for the $25 \mathrm{~nm}$ device. Note the different dependences on $V_{D}$.

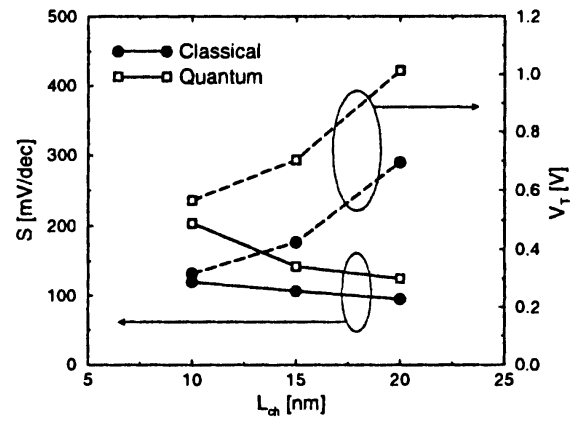

Figure 8: Short-channel performance of nanoscale MOSFET's as a function of the metallurgical channel length.

$S$ changes from $107 \mathrm{mV} /$ decade to $143 \mathrm{mV} /$ decade for classical and QM simulations, respectively. This very high increase (about $33 \%$ ) will significantly offset the traditional MOS scaling scenario and will accelerate the adoption of innovative structures.

QM effects on the short-channel performances have been also investigated. The offstate current of the device is shown in Fig. 7 as a function of $V_{D}$. As the computational time rapidly increases for high $V_{D}$, QM simulations were limited to $V_{D}=0.2 \mathrm{~V}$. The off current is reduced in the QM model due to the increased $V_{T}$. However, DIBL performances are degraded due to the source-to-drain tunneling contribution to the current. Fig. 8 shows $V_{T}$ and $S$ as functions of the metallurgical channel length. Comparable $V_{T}$ roll-offs are obtained, meaning that the $\mathrm{QM}$ charge distribution does not influence the barrier lowering in the channel. Significant degradation in the subthreshold slope is instead obtained in the QM simulations, in agreement with the results in Fig. 7.

\section{Conclusions}

We have developed a fully 2D QM model for simulation of nanoscale MOSFET's. A semiclassical transport model is adopted, supported by comparison against a $\mathrm{QM}$ model. Simulation results show that $\mathrm{QM}$ effects strongly influence important device performances such as threshold voltage, subthreshold slope, DIBL and SCE. We conclude that full QM simulations will become a mandatory issue for nanoscale MOSFET's modeling and design.

\section{References}

[1] Chen, W., Ouyang, Q., Register, L. F., Banerjee, S. K. (2000): Quantum effects along the channel of ultra-scaled Si-based MOSFETs?. In: IEDM 2000 Tech. Dig., pp. 291-294

[2] Fischetti, M. V. (1998): Theory of electron transport in small semiconductor devices using the Pauli master equation. J. Appl. Phys. 83: 270-291

[3] Lent, C. S., Kirkner, D. J. (1990): The quantum transmitting boundary method. J. Appl. Phys. 67: 6353-6359

[4] Semiconductor Industry Assoc. (1999): International technology roadmap for semiconductors [5] Spinelli, A., Benvenuti, A., Pacelli, A. (1998): Self-consistent 2D model for quantum effects in n-MOS transistors. IEEE Trans. Electron Devices 45: 1342-1349 